\title{
Delineating the extent of anterior capsulorhexis with gentian violet using capsulorhexis marker: a preliminary study of efficacy and toxicity in an animal model
}

Mohamed Abdel Moneim

Hassaballa

Amr Abd el-Latif Osman

Department of Ophthalmology,

Faculty of Medicine, Cairo University, Cairo, Egypt
Correspondence: Mohamed Abdel Moneim Hassaballa

10 Madkour St, El Haram, Giza, Egypt

Tel +20 20I 01680505

Emailmhassaballal@gmail.com
This article was published in the following Dove Press journal:

Clinical Ophthalmology

16 June 2011

Number of times this article has been viewed

Background: The purpose of this study was to evaluate the efficacy and safety of a new instrument to optimize the size, shape, and centration of anterior capsulorhexis.

Methods: The study was divided into two parts. The first part was done in 10 goats' eyes. A specially designed capsulorhexis marker was used. The lower edge of the instrument was stained by gentian violet and then applied to the anterior capsule to produce a circular mark of $5.5 \mathrm{~mm}$. In five eyes, the gentian violet was applied to the marker using a corneal marking pen; in the remaining five eyes, the gentian violet was applied using a corneal marking pad. In the second part, the toxicity of gentian violet was tested as follows: ten albino rabbits received a gentian violet injection into the anterior chamber in one eye (experimental group) and an equal volume of balanced salt solution in the fellow eye (control group). Five rabbits were sacrificed one day after surgery and the remaining five rabbits after one week.

Results: In the first part, there was no difficulty in the introduction or removal of the instrument from all eyes. In the first two eyes, the circular mark was diffuse due to sliding of the marker on the surface of the anterior capsule. In the remaining eight eyes, there was a well-centered, rounded mark which was adequately stained. In the second part, there was no histopathological evidence of corneal toxicity in either group. There was loss of ganglion cells from the neurosensory retina one day after surgery in one eye from the experimental group. At one week, there was no evidence of retinal toxicity in any of the rabbits.

Conclusion: This capsulorhexis marker can guide the surgeon to a better centration and proper sizing of anterior capsulorhexis using gentian violet staining. More refinement of the instrument is needed to be able to use it in human eyes.

Keywords: capsulorhexis, gentian violet, marker

\section{Introduction}

With the introduction of phacoemulsification as standard cataract surgery, anterior capsulorhexis has become an essential step. Anterior capsulorhexis can be performed using a 26-gauge needle or capsulorhexis forceps. ${ }^{1,2}$ However, when performed free-hand, it is not always easy to create a perfectly sized anterior capsulorhexis that is also perfectly centered relative to the pupil or the limbus. Although the anterior capsulorhexis obtained is usually continuous, it may still be noncurvilinear, varying from kidney-shaped to heart-shaped, and in some cases it may be discontinuous. ${ }^{3}$ Moreover, the introduction of multifocal intraocular lenses has increased the need for better surgical performance and precision. 
Many investigators have attempted to design new tools for better control of the size and shape of the capsulorhexis, such as diathermy rhexis ${ }^{4}$ and vitrectorhexis. ${ }^{5}$ Certain styles of capsule forceps have also been designed with laser marks to help surgeons produce various capsulotomy diameters. However, these forceps are limited in their ability to provide a diameter reference in the horizontal meridian parallel to the phacoemulsification incision. ${ }^{6}$

The aim of this study was to evaluate the efficacy and safety of a new instrument to optimize the size, shape, and centration of anterior capsulorhexis during phacoemulsification by making a circular mark on the anterior capsule using gentian violet stain.

\section{Materials and methods}

This study was divided into two parts. The first part was performed in a fully equipped wet laboratory (Alcon Laboratories, Cairo, Egypt) where goats' eyes were used. The second part was done at Cairo University Medical School animal house facility using albino rabbits. We adhered to the tenets of the National Institutes of Health statement on the use of animals in research.

\section{Use of capsulorhexis marker to stain the anterior capsule}

A stainless steel capsulorhexis marker was specially designed for this study. It was formed of a handle attached to a ring with an inner diameter of $5.5 \mathrm{~mm}$ and a height of $1 \mathrm{~mm}$ (Figure 1). Ten goats' eyes were prepared for the procedure. A $3 \mathrm{~mm}$ corneal tunnel was performed in each eye followed by injection of viscoelastic material into the anterior chamber. The wound was then widened to $6 \mathrm{~mm}$ to allow easy introduction of the instrument. In five eyes, the lower edge of the ring was stained by gentian violet using a surgical marking pen (Viscot Medical, East Hanover, NJ) whereas in the other five eyes, the lower edge

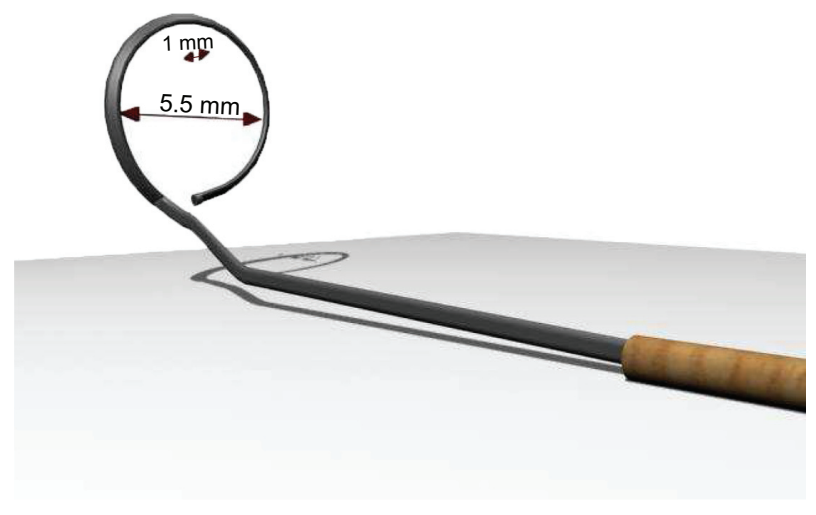

Figure I Diagrammatic representation of capsulorhexis marker. of the ring was stained by gentian violet using a surgical marker

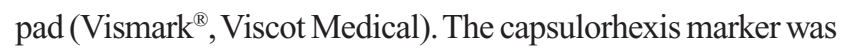
introduced into the anterior chamber and the ring was centered within the papillary area. The lower edge of the ring was applied onto the anterior capsule for a few seconds without pressure to avoid zonular stress. The marker was then removed from the eye and assessment of the mark was done as regards centration and degree of staining of the anterior capsule (Figure 2).

\section{Assessment of intraocular toxicity}

Because we were using a preparation of gentian violet designed for extraocular use (on the corneal surface), we needed to assess the intraocular toxicity in an animal model. Accordingly, 10 albino rabbits weighing $2-4 \mathrm{~kg}$ received a gentian violet injection into the anterior chamber in one eye (experimental group), and an equal volume of balanced salt solution in the fellow eye (control group).

\section{Surgical technique}

The rabbits were anesthetized with an intramuscular injection of xylazine 5-8 mg/ $/ \mathrm{kg}$ and ketamine hydrochloride $35-44 \mathrm{mg} /$ $\mathrm{kg}$. The pupils were dilated with tropicamide and phenylephrine $10 \%$ eye drops. In the experimental group, the lower edge of the ring was stained by gentian violet using the surgical marking pen. The ring was then left in the well of a sterile contact lens case containing balanced salt solution $0.1 \mathrm{~mL}$ for two minutes. The stained balanced salt solution was then injected into the anterior chamber using a 27 -gauge needle. In the control group, balanced salt solution $0.1 \mathrm{~mL}$ was injected

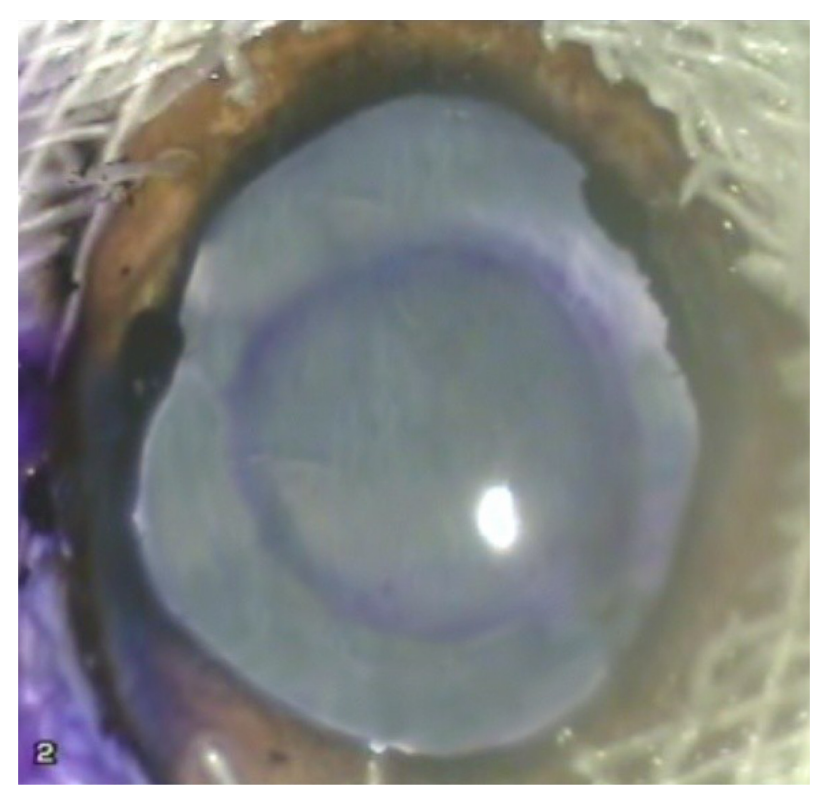

Figure 2 Capsulorhexis mark on the anterior capsule. 
into the anterior chamber using a 27-gauge needle. All animals were kept in the animal house and were handled according to the Association for Research in Vision and Ophthalmology statement on use of animals in ophthalmic and vision research. Five rabbits were sacrificed one day after surgery and the remaining five after one week. The eye balls were enucleated and immediately fixed in Bouin's solution, dehydrated in ascending grades of alcohol $(70 \%, 90 \%$, and $100 \%)$, cleared in xylol, and embedded in paraffin. The embedded specimens were then sectioned (5-7 $\mu \mathrm{m}$ thickness), stained with hematoxylin and eosin, and examined by light microscopy.

\section{Results}

\section{Use of capsulorhexis marker to stain anterior capsule}

There was no difficulty in the introduction or removal of the instrument from any of the eyes. There was no difference in the degree of capsular staining using either the marker pen or the marker pad. In the first two eyes, the mark was not clear due to sliding of the ring on the surface of the anterior capsule; this resulted in spread of the stain, leading to a diffuse mark. In all other cases, care was taken not to touch the anterior capsule during introduction of the instrument into the anterior chamber. Once in place, the ring was pressed down on the anterior capsule without undue sliding. This resulted in a well-centered rounded mark which was adequately stained, precise, and clear enough to guide the surgeon to perform a $5.5 \mathrm{~mm}$ capsulorhexis.

\section{Assessment of intraocular toxicity}

Histopathologically, none of the eyes in the study or control group showed any sign of corneal toxicity at one day and one

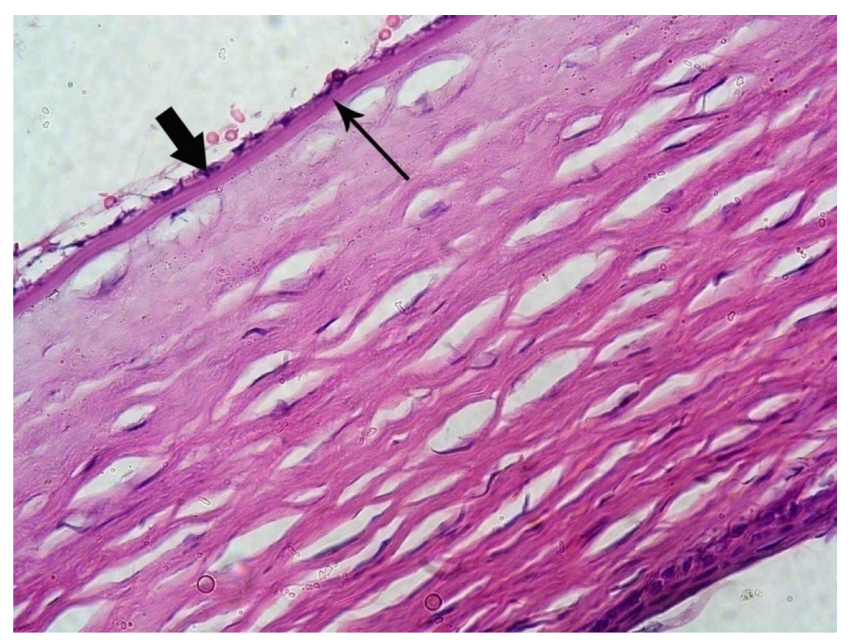

Figure 3 Photomicrograph of a control rabbit cornea showing flat endothelial cells with flat nuclei (thick arrow) resting on Descemet's membrane (thin arrow). Hematoxylin and eosin staining, 400x.

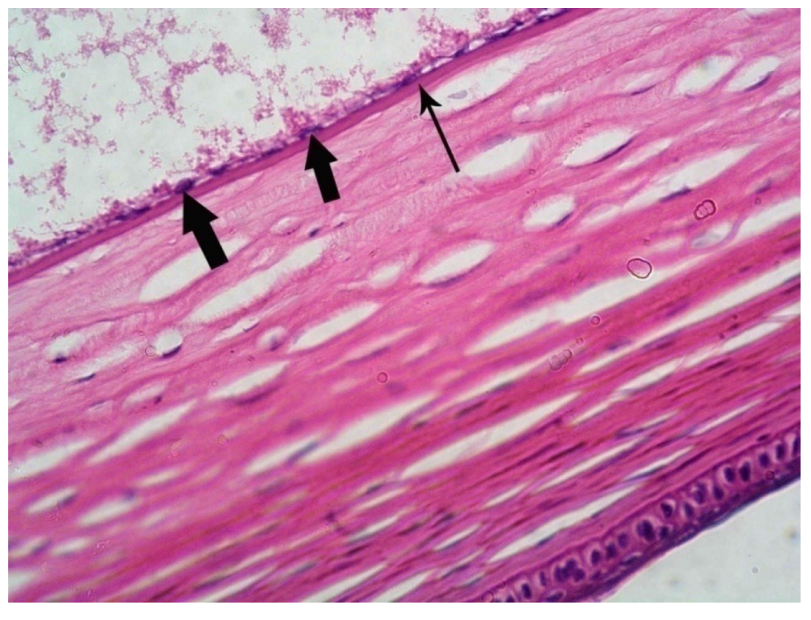

Figure 4 Photomicrograph of an experimental rabbit's cornea showing normally appearing flat endothelial cells (thick arrow) and intact Descemet's membrane (thin arrow). Hematoxylin and eosin staining, 400x.

week after surgery (Figures 3 and 4). Light microscopy examination of multiple sections from each eye showed normal iris tissue, ciliary body epithelium, and lens epithelium. The retina was normal in all eyes of the control group at one day and one week after the surgery (Figure 5). In the experimental group, there was no evidence of retinal toxicity at one day and one week after surgery with the exception of one eye that showed evidence of loss of ganglion cells from the neurosensory retina one day after surgery (Figure 6).

\section{Discussion}

A well constructed capsulorhexis is the foundation of complication-free phacoemulsification and intraocular lens surgery. Ophthalmic surgical dyes have become valuable tools, and are now widely used in both anterior and posterior segment

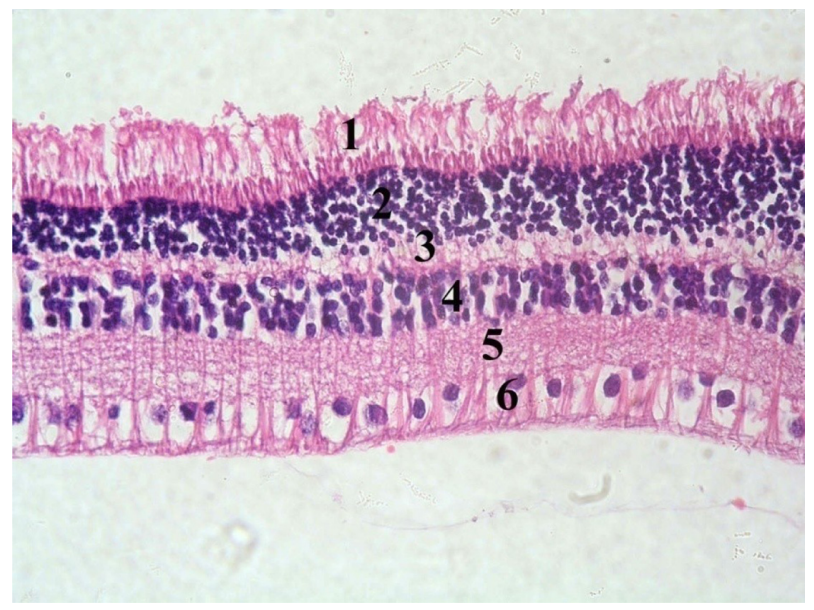

Figure 5 Photomicrograph of a section in a control rabbit's retina showing photoreceptors layer (1), outer nuclear layer (2), outer reticular layer (3), inner nuclear layer (4), inner reticular layer (5), and ganglion cell layer (6). Hematoxylin and eosin staining, 400x. 


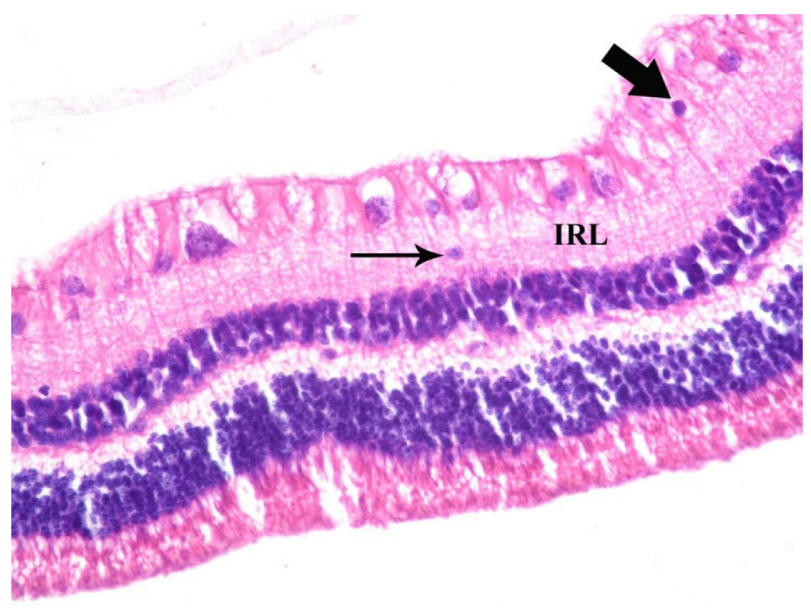

Figure 6 A photomicrograph of a section in an experimental rabbit's retina showing a ganglion cell with pyknotic (dark) nucleus (thick arrow) and another one (thin arrow) that is displaced within the inner reticular layer (IRL). Hematoxylin and eosin staining, $400 \times$.

indications. In this study, we present a preliminary prototype of a capsulorhexis marker which is first stained by gentian violet and then applied directly onto the anterior capsule in order to make a rounded mark to guide the surgeon to a better centering and proper sizing of the rhexis. Moreover, it may help phacoemulsification beginners and shorten their learning curve for this crucial step in modern cataract surgery.

Today's demanding refractive results require a wellcentered, perfectly circular capsulorhexis that slightly overlaps the intraocular lens optic. This construction is important for achieving the so-called capsular "shrink-wrap" effect during the postoperative period. A $360^{\circ}$ overlapping capsular edge creates a capsular bend, which acts as a barrier against proliferating lens epithelial cells and thus significantly delays the onset of posterior capsular opacification..$^{7-9}$ This overlap also sets the anteroposterior positioning of the intraocular lens, which prevents capsular fibrosis from shifting the lens optic forward and creating an unwanted late refractive shift. Moreover, with a perfectly circular capsulorhexis, any contraction of the anterior capsule (ie, phimosis) will be symmetrical, so late in-the-bag decentering of the optic is prevented. ${ }^{10}$ Also, if the capsulorhexis is too small, it can result in glare, halos, and night driving problems with a diffractive multifocal intraocular lens. Capsular phimosis is more pronounced with small capsulotomies. ${ }^{11,12} \mathrm{~A}$ small capsular opening makes surgery more difficult and places greater stress on the anterior capsule during nuclear division or quadrant removal. Furthermore, a smaller capsular opening can obscure peripheral retinal visibility and treatment. A capsulorhexis with a $5.5 \mathrm{~mm}$ diameter is usually ideal.
Many investigators have tried to improve the predictability of size, shape, and centration of capsulorhexis. Wallace ${ }^{6}$ performed a study to make a central corneal mark with a $6.0 \mathrm{~mm}$ optical zone marker, previously used for radial keratotomy (with the center of the optical zone as the central visual axis), as the patient fixates on the light of the microscope. He performed the capsulorhexis in the usual way with the intention of leaving the borders of the capsulotomy inside the capsulotomy diameter mark. However, this technique has some disadvantages. First, because corneal curvatures are not uniform between patients, a different mark size would be required for each patient to achieve the correct capsulorhexis size. Moreover, use of this marker incorrectly implies that corneal curvature remains constant after the incisions are made. ${ }^{3}$ Finally, the corneal plane and the anterior capsule plane must be parallel with the focal plane of the operating microscope to avoid parallax errors, and the surgeon may confuse the corneal mark with the anterior capsule rim at the time of intraocular lens implantation. ${ }^{13}$

Tassignon et $\mathrm{al}^{3}$ designed a $0.25 \mathrm{~mm}$ ring-shaped caliper with an internal diameter of $5.0 \mathrm{~mm}$ or $6.0 \mathrm{~mm}$. After its insertion, the ring is gently pushed on top of the anterior capsule with additional Healon GV. A small opening is made in the center of the capsule. The surgeon then carefully follows the internal border of the ring caliper. However, this technique is not precise because the ring is not fixed on the anterior surface of the capsule, and thus any movement of the ring will affect the size, shape, and centration of the capsulorhexis.

A promising technological solution involves overlaying a reference ring on the surgeon's view through the microscope. Carl Zeiss Meditec Inc developed an interface module that adjusts the size of the ring appropriately with microscope magnification while keeping it centered within the limbus using real-time eye tracking. ${ }^{14}$ Moreover, several companies are developing femtosecond technologies to perform the capsulorhexis.

To our knowledge, this is the first published article in the literature describing an instrument that makes a circular mark on the anterior capsule using a stain. Gentian violet (hexamethyl rosaniline chloride) is a triphenylmethane (rosaniline) dye. The pure compound is known as crystal violet. It was chosen for this study because it is cheap, easily available, and intensely and homogeneously stains the anterior capsule. ${ }^{15}$

Different techniques have been proposed for staining of the anterior capsule by different dyes. Many surgeons continue to inject capsular dye beneath an air bubble, as originally 
described by Horiguchi et al ${ }^{16}$ and Melles et al. ${ }^{17}$ Laureano and Coroneo ${ }^{18}$ suggested simply omitting the air bubble. Kayikicioglu et $\mathrm{al}^{19}$ have used a viscoelastic-mixing technique with $0.4 \%$ trypan blue. In this study, we applied the dye directly onto the anterior surface of the capsule by the lower edge of the capsulorhexis marker and this helped in decreasing the amount of the dye inserted into the anterior chamber and also in avoiding inadvertent application of the dye towards the corneal endothelium.

Many studies have investigated the ocular toxicity of gentian violet. Previously, gentian violet $0.1 \%$ with methylene blue $1 \%$ was used for staining. ${ }^{17}$ This combination is no longer used because the endothelial toxicity of the dyes causes corneal edema. Ünlü et $\mathrm{al}^{15}$ examined the effects of gentian violet $0.001 \%$ and gentian violet $0.01 \%$ without methylene blue and found that each concentration of gentian violet alone was effective in staining the anterior capsules of white cataracts and found that both concentrations had no toxic effects on intraocular tissues. Gamal Eldin et $\mathrm{a}^{20}$ reported that gentian violet $0.05 \%-2 \%$ stained the anterior capsule of the rabbit eye effectively, but that only the lowest concentration $(0.05 \%)$ showed excellent preservation of the cornea during one week of observation. In the second part of our study, we studied the toxicity of gentian violet derived from the marker pen. This pen is generally used to make an external mark on the outer surface of the cornea during keratoplasty and keratorefractive procedures. No toxic changes were evident in the cornea, iris tissue, ciliary body epithelium, or in the lens epithelium. Only one eye had partial ganglion cell loss from the retina one day after surgery. This may represent a preparation artifact rather than a true toxic effect.

In this study, we used a simple preliminary prototype that needs a large corneal incision $(6 \mathrm{~mm})$ to be introduced inside the eye. We considered this as the first step in our research and we are looking forward to changing the material of the marker in order to form a memory ring that is able to be introduced and removed from the eye through a small incision and become ergonomically feasible. However, it can be used as such in cases of combined cataract extraction with penetrating keratoplasty because there is enough room to introduce the instrument after trephination of the recipient cornea. An important issue to be addressed is the proper way to centralize the marker on the surface of the anterior capsule in relation to the visual axis.

\section{Conclusion}

This capsulorehxis marker is an instrument designed to optimize the size, shape, and centering of the capsulorhexis during phacoemulsification by impressing a circular pattern on the anterior capsule to guide the surgeon in creating a 5.5-6.0 mm diameter capsulorhexis. More refinement of the instrument is needed to be able to use it in human eyes.

\section{Acknowledgment}

We express our deep gratitude to Dr Dina Helmy for her help and support in preparation and interpretation of the histopathologic specimens in the second part of the study. We also express our appreciation to Alcon Egypt for allowing use of its wet laboratory facility in the first part of the study.

\section{Disclosure}

The authors report no conflicts of interest in this work.

\section{References}

1. Zamini M, Buratto L, Savin G. Capsulorhexis. In: Buratto L, Werner L, Zanini M, Apple D, editors. Phacoemulsification: Principles and Techniques. Thorafore, NJ: Slack Incorporated; 2003.

2. Neuhan TF. Capsulorhexis. In: Steinert RF, Fine H, Gimbel HV, et al. editors. Cataract Surgery. New York, NY: Elsevier Science; 2004.

3. Tassignon MJ, Rozema J, Gobin L. Ring-shaped caliper for better anterior capsulorhexis sizing and centration. J Refract Surg. 2006;32:1253-1255

4. Shohei F, Masayuki M, Kiyoshi S, et al. Morphological changes in cataract anterior capsule in diathermy capsulorhexis using different types of viscoelastic agents. Journal of the Eye. 2001;18: 795-799.

5. Hazirolan DO, Altiparmak UE, Aslan BS, Duman S. Vitrectorhexis versus forceps capsulorhexis for anterior and posterior capsulotomy in congenital cataract surgery. J Pediatr Ophthalmol Strabismus. 2009;46:104-107.

6. Wallace R. Capsulotomy diameter mark. J Refract Surg. 2003;29: $1866-1868$

7. Apple DJ, Peng Q, Visessook N, et al. Eradication of posterior capsule opacification: documentation of a marked decrease in Nd:YAG laser posterior capsulotomy rates noted in an analysis of 5416 pseudophakic human eyes obtained postmortem. Ophthalmology. 2001;108:505-518.

8. Aykan U, Bilge AH, Karadayi K. The effect of capsulorhexis size on development of posterior capsule opacification: small (4.5 to $5.0 \mathrm{~mm})$ versus large (6.0 to $7.0 \mathrm{~mm}$ ). Eur J Ophthalmol. 2003;13:541-545.

9. Hollick EJ, Spalton DJ, Meacock WR. The effect of capsulorhexis size on posterior capsular opacification: one-year results of a randomized prospective trial. Am J Ophthalmol. 1999;128:271-279.

10. Altmann GE, Nichamin LD, Lane SS, Pepose JS. Optical performance of 3 intraocular lens designs in the presence of decentration. J Cataract Refract Surg. 2005;31:574-585.

11. Sugimoto Y, Takayanagi K, Tsuzuki S, et al. Postoperative changes over time in size of anterior capsulorhexis in phacoemulsification/aspiration. Jpn J Ophthalmol. 1998;42:495-498.

12. Joo CK, Shin JA, Kim JH. Capsular opening contraction after continuous curvilinear capsulorhexis and intraocular lens implantation. J Cataract Refract Surg. 1996;22:585-590.

13. Kellen R. Capsulotomy diameter mark. J Refract Surg. 2004;30(10): 2031-2032.

14. Dick HG, Pena-Aceves A, Manns M, Krummenauer F. New technology for sizing the continuous curvilinear capsulorhexis: prospective trial J Cataract Refract Surg. 2008;34:1136-1144. 
15. Ünlü K, Askünger A, Söker S, et al. Gentian violet solution for staining the anterior capsule. J Cataract Refract Surg. 2000;26:1228-1232.

16. Horiguchi M, Miyake K, Ohta I, Ito Y. Staining of the lens capsule for circular continuous capsulorhexis in eyes with white cataract. Arch Ophthalmol. 1998;116:535-537.

17. Melles G, de Waard P, Pameyer J, Beekhuis W. Trypan blue capsule staining to visualize the capsulorhexis in cataract surgery. $J$ Cataract Refract Surg. 1999;25:7-9.
18. Laureano JS, Coroneo MT. Crystalline lens capsule staining with trypan blue. J Cataract Refract Surg. 2004;30:2046-2049.

19. Kayikicioglu O, Erakgun T, Guler C. Trypan blue mixed with sodium hyaluronate for capsulorhexis. J Cataract Refract Surg. 2003;27:970.

20. Gamal Eldin SA, El Mehelmy EM, El Shazli EM, Mostafa YMS. Experimental staining of the anterior lens capsule in albino rabbits. J Cataract Refract Surg. 1999;25:1289-1294.

\section{Publish your work in this journal}

Clinical Ophthalmology is an international, peer-reviewed journal covering all subspecialties within ophthalmology. Key topics include: Optometry; Visual science; Pharmacology and drug therapy in eye diseases; Basic Sciences; Primary and Secondary eye care; Patient Safety and Quality of Care Improvements. This journal is indexed on

\section{Dovepress}

PubMed Central and CAS, and is the official journal of The Society of Clinical Ophthalmology (SCO). The manuscript management system is completely online and includes a very quick and fair peer-review system, which is all easy to use. Visit http://www.dovepress.com/ testimonials.php to read real quotes from published authors. 